\title{
Multi-Detector-Row CT Diagnosis of Adrenal Incidentaloma in Patients with Hepatocellular Carcinoma
}

\author{
Taisuke Harada $^{1}$, Tamotsu Kamishima ${ }^{2}$, Satoshi Terae ${ }^{1}$, Yuya Onodera ${ }^{3}$, Hiroki Shirato ${ }^{4}$ \\ ${ }^{1}$ Department of Diagnostic and Interventional Radiology, Hokkaido University Hospital, Sapporo City, Japan \\ ${ }^{2}$ Faculty of Health Science, Hokkaido University, Sapporo City, Japan \\ ${ }^{3}$ Department of Radiology, Keiyukai Sapporo Hospital, Sapporo City, Japan \\ ${ }^{4}$ Department of Radiation Medicine, Hokkaido University, Graduate School of Medicine, Sapporo City, Japan \\ Email: ktamotamo2@yahoo.co.jp
}

Received November 27, 2012; revised December 21, 2012; accepted January 4, 2013

\begin{abstract}
We investigate the diagnostic reliability of differentiating between lipid-poor adrenal adenomas and metastatic adrenal tumors originating from hepatocellular carcinoma (HCC) using a routine dynamic CT protocol for liver imaging. Eighteen metastatic adrenal tumors originating from HCC and 13 lipid-poor adrenal adenomas were identified. Dynamic CT data were analyzed for CT attenuation of adrenal lesions before and after contrast administration. When a cutoff of $36 \mathrm{HU}$ was set for adrenal lesions at pre-contrast attenuation, the sensitivity and specificity for the diagnosis of metastatic lesions were $94.4 \%$ and $92.3 \%$, respectively. Attenuation criteria on pre-contrast CT may help optimize the differentiation between these lesions.
\end{abstract}

Keywords: CT; Adrenal Incidentaloma; Hepatocellular Carcinoma; Lipid-Poor Adrenal Adenomas; Metastatic Adrenal Tumors

\section{Introduction}

Hepatocellular carcinoma (HCC) results in between 250,000 and one million deaths globally per year [1-4]. Hepatocellular carcinoma is a devastating tumor, with a mean survival time of much less than 1 year, if left untreated [5]. The treatment of HCC depends entirely on tumor stage. Potentially curative partial hepatic resection or orthotopic liver transplantation should be reserved for patients with stage I or II tumors [6]. Patients with an advanced tumor staging or with poor hepatocellular reserves are not candidates for these curative surgical treatments, and therefore have palliative care as their only option. Patients with advanced-stage disease have lower survival rates than patients with early-stage HCC tumors. Thus, the detection of extrahepatic metastatic disease becomes a crucial factor in planning potential therapy for patients with HCC, as it is desirable to avoid unnecessary surgical interventions. The adrenal glands are a common site for extrahepatic metastases, which accounted in one study for $19(8.4 \%)$ of 232 consecutive cases of hepatocellular carcinoma autopsied [7].

Recent studies have predicted that an adrenal incidentaloma will be identified in $4 \%-5 \%$ of abdominal CTs [8]. However approximately $75 \%$ of adrenal masses in patients with no history of cancer are cortical adenomas, whereas adrenal metastases account for $45 \%-73 \%$ of adrenal masses in cancer patients [8]. Computed tomography has become the imaging modality of choice to differentiate between benign adrenal adenomas and metastases in oncology patients [9]. As approximately $30 \%$ of adenomas are lipid-poor and difficult to characterize on unenhanced CT scans [10], it has been proposed that if the attenuation of the adrenal gland exceeds $10 \mathrm{HU}$ upon non-enhanced CT, contrast material-enhanced CT should be performed, and the washout calculated. With a combination of unenhanced and delayed enhanced CT, nearly all adrenal masses can be correctly categorized as adenomas or non-adenomas [11,12].

In patients with $\mathrm{HCC}$, adrenal incidentalomas may be depicted if the patient is undergoing dynamic CT for an HCC protocol that would include the hepatic arterial, portal, and equilibrium phases after contrast administration, in addition to non-enhanced CT. Lipid-rich adrenal adenomas may be diagnosed by analyzing unenhanced $\mathrm{CT}$ images. On the other hand, it remains difficult to differentiate metastatic adrenal tumors from lipid-poor adrenal adenomas via CT images using an HCC protocol without a 10-minute delayed CT scan. As regards the CT-imaging results of adrenal tumors metastasized from HCC, Katyal and colleagues reported finding that contrast material-enhancement characteristics ranged from the typical hypoattenuation of soft tissue (seen in adrenal 
metastases from other primary tumors) to a marked hyperattenuating enhancement [13]. However, to the best of our knowledge, there has been no detailed analysis of the attenuation value of adrenal tumors metastasized from HCC as observed on dynamic CT. We hypothesized that it would be possible to differentiate between metastatic adrenal HCC lesions and lipid-poor adenomas using a $\mathrm{CT}$ protocol that is routinely performed for patients with HCC. Therefore, the purpose of this study was to investigate the diagnostic value of a routine HCC imaging protocol to differentiate between lipid-poor adenomas and metastatic adrenal tumors originating from HCC.

\section{Materials and Methods}

\subsection{Ethical Issues}

We adapted the requirements of our institutional review board for a retrospective observation study, and the requirement for informed consent was waived.

\subsection{Patient Selection}

Diagnostic reports from dynamic CT examinations performed between January 2002 and December 2009 that contained the words "hepatocellular carcinoma", "adrenal adenoma", or "adrenal metastasis" were searched retrospectively. In 2888 reports identified, the diagnosis of adrenal metastasis was based on rapid growth (more than $50 \%$ growth in the largest diameter) of a mass in 6 months. Metastases exceeding $30 \mathrm{~mm}$ in the largest diameter were excluded, because adrenal adenomas larger than $30 \mathrm{~mm}$ are uncommon. Cases involving malignant tumors in organs other than the liver were excluded to prevent inclusion in the analysis of any confounding data from metastatic tumors that had originated in other organs. When there were multiple follow-up studies of metastatic HCC patients, the CT study (usually the latest) involving the largest tumor with a diameter of less than $30 \mathrm{~mm}$ was selected for analysis. A diagnosis of adenoma was established on the basis of stable size of the mass for more than half a year. Adrenal adenomas of less than $5 \mathrm{~mm}$ in the largest diameter were excluded to avoid partial volume averaging with densitometry. Lipid-rich adenomas with an average $\mathrm{CT}$ number of less than 20 HU were excluded, because the study was designed with a focus on lipid-poor adenomas. In our selection of lipidpoor adenomas, adrenal nodules with cysts, macroscopic fat, or calcifications were also excluded. We identified 12 patients ( 8 men, 4 women; age range: 57 - 81 years; mean age: 68.4 years) with 13 lipid-poor adenomas, and 15 patients (12 men, 3 women; age range: 41 - 80 years; mean age: 61.1 years) with 18 adrenal metastases from HCC. In 10 cases of metastases, histological proof was available, and 4 cases were bilateral.

\subsection{CT scan}

Dynamic CT scans were performed with patients in the supine position. Breath-hold CT examinations of the abdomen were performed with a 4-section or a 64-section multi-detector Aquilion Scanner (Toshiba Medical Systems Corporation, Otawara, Tochigi, Japan). The following scan parameters were used for the 4-section multi-detector scanner: tube current, $140 \mathrm{mAs}$; voltage, 120 $\mathrm{kV}$; collimation, $4 \times 2 \mathrm{~mm}$; reconstructed slice thickness, $5 \mathrm{~mm}$; and reconstruction increment, $5 \mathrm{~mm}$. The following scan parameters were used for the 64-section multidetector scanner: tube current-via automatic exposure control; voltage, $120 \mathrm{kV}$; collimation, $64 \times 0.5 \mathrm{~mm}$; reconstructed slice thickness, $5 \mathrm{~mm}$; and reconstruction increment, $5 \mathrm{~mm}$. Scans of the abdomen were performed in the craniocaudal direction with a protocol that included the hepatic arterial phase (approximately $40 \mathrm{sec}-$ onds after the onset of contrast injection) and equilibrium phase (150 seconds after the onset of contrast injection), with the scan timing adjusted by a bolus tracking system. The dosage of the contrast material was $450 \mathrm{mgI} / \mathrm{kg}$ (body weight) of iodine contrast agent. It is important to emphasize that the fixed injection duration of 30 seconds, used for the present injection protocol, was selected over a fixed injection rate in order to establish an optimal contrast-enhanced dynamic CT protocol of the liver; in general, injection duration is considered the most important of the two measures, and is the only technical factor capable of predicting the scan timing for each phase [14].

\subsection{Image Analysis}

Computed tomographic images were retrieved from the institutional picture archiving and communication system (PACS) to a standard viewer (Vox-Base; J-Mac Systems, Sapporo, Japan). Average attenuation values on the precontrast, hepatic arterial contrast-enhanced, and equilibrium contrast-enhanced images were recorded. A circular region of interest (ROI) was placed in the center of the adrenal mass, in a section where the mass appeared largest; the region of interest covered approximately one-half to two-thirds of the mass. Care was taken to avoid the partial-volume effect and beam-hardening artifacts. All images were retrospectively reviewed by a radiologist (HT) with 2 years of training in abdominal imaging. When more than one adrenal mass was present, all measurements were obtained for each mass. We determined both the relative percentage washout (RPW) and absolute percentage washout (APW) rates. The RPW and APW were calculated as follows: $\mathrm{RPW}=100 \cdot(\mathrm{HA}-\mathrm{EqA}) / \mathrm{HA}$ and $\mathrm{APW}=100 \cdot([\mathrm{HA}-\mathrm{EqA}] /[\mathrm{HA}-\mathrm{PA}])$, where $\mathrm{HA}$ is attenuation on hepatic arterial contrast-enhanced scans, EqA is attenuation on equilibrium contrast-enhanced scans, PA is precontrast attenuation, and all attenuation meas- 
urements are in Hounsfield units.

\subsection{Statistical Analysis}

Statistical analysis was performed using MedCalc statistical software, version 7.2. 0.2 (MedCalc Software, Mariakerke, Belgium). Quantitative variables are given as the average and range. The independent t-test was performed to assess differences in values. To determine optimal cutoffs and calculate the area under the curve (AUC), sensitivity and specificity, and receiver operating characteristics curve (ROC) analyses were performed. Any $\mathrm{p}$ value less than 0.05 was considered statistically significant.

\section{Results}

Detailed data on the size, laterality, CT attenuation (precontrast CT, hepatic arterial phase, and equilibrium phase), APW, and RPW for the lipid-poor adenoma and metastasis groups are shown in Table 1. Size, CT at- tenuation, and washout percentage were all significantly larger in the HCC group than in the adenoma group, with the exception of the CT attenuation on the arterial phase. Figure 1 shows the scatterplot distribution of the precontrast attenuation values of lipid-poor adenomas and metastases. When we set the cutoff value at $36 \mathrm{HU}$, sensitivity and specificity were $94.4 \%$ and $92.3 \%$, respectively. The results and a comparison ROC analysis for differentiating between the two groups are shown in Table 2 and Figure 2. Precontrast CT attenuation showed the largest AUC value of 0.97 . Out of 18 nodules classified as metastatic adrenal tumors, histopathological analysis was possible in 10 cases, 8 tumors were diagnosed as predominantly moderately differentiated $\mathrm{HCC}$ with 2 of these 8 cases having a poorly differentiated component, and with 1 case of well-differentiated component. Two tumors were diagnosed as poorly differentiated HCC. Both typical and atypical examples of images of lipidpoor adenomas and metastases are shown in Figures 3-6.

Table 1. Size, laterality, CT attenuation (pre-contrast CT, hepatic arterial phase, and equilibrium phase), APW, and RPW for lipid-poor adenomas and adrenal metastases originating from HCC.

\begin{tabular}{|c|c|c|c|}
\hline & Lipid-poor adenoma $(\mathrm{n}=13)$ & $\operatorname{HCC}(\mathrm{n}=18)$ & P-value \\
\hline Age & $69.4 \pm 8.3(57$ to 81$)$ & $61.1 \pm 8.7(41$ to 80$)$ & 0.0120 \\
\hline Laterality & $\mathrm{L}: \mathrm{R}=9: 4$ (bilateral 1) & $\mathrm{L}: \mathrm{R}=9: 9$ (bilateral 4) & 0.2843 \\
\hline Size $(\mathrm{mm})$ & $14.1 \pm 4.3(8$ to 24$)$ & $22.3 \pm 5.4(14$ to 24$)$ & $<0.0001$ \\
\hline \multicolumn{4}{|l|}{ Attenuation on $\mathrm{CT}$} \\
\hline Precontrast CT (HU) & $29.0 \pm 6.4(21.3$ to 41.4$)$ & $48.1 \pm 8.5(35.4$ to 64.6$)$ & $<0.0001$ \\
\hline Arterial phase (HU) & $80.7 \pm 25.5(29.9$ to 117.5$)$ & $86.0 \pm 20.9(52.5$ to 120.6$)$ & 0.5295 \\
\hline Equilibrium phase (HU) & $53.8 \pm 16.6(25.2$ to 71.6$)$ & $74.7 \pm 13.1(59.2$ to 107.9$)$ & 0.0005 \\
\hline APW (\%) & $51.6 \pm 24.5(7.9$ to 87.7$)$ & $6.16 \pm 68.6(-237.3$ to 59.3$)$ & 0.0304 \\
\hline RPW (\%) & $30.0 \pm 18.1(3.7$ to 60.4$)$ & $10.6 \pm 14.8(-14.8$ to 32.5$)$ & 0.002 \\
\hline
\end{tabular}

The RPW and APW were calculated as follows: RPW $=100 \cdot(\mathrm{HA}-\mathrm{EqA}) / \mathrm{HA}$ and APW $=100 \cdot([\mathrm{HA}-\mathrm{EqA}] /[\mathrm{HA}-\mathrm{PA}])$, where HA is attenuation on hepatic arterial contrast-enhanced scans, EqA is attenuation on equilibrium contrast-enhanced scans, PA is pre-contrast attenuation, and all attenuation measurements are in Hounsfield units (HU).

Table 2. Receiver operating characteristics (ROC) analysis for differentiating between lipid-poor adenomas and adrenal metastases originating from HCC.

\begin{tabular}{ccccc}
\hline & AUC & Standard error & 95\% confidence interval & Significance level \\
\hline Size (mm) & 0.868 & 0.0713 & 0.682 to 0.966 & 0.0001 \\
Attenuation on CT & & & & 0.836 to 0.994 \\
Pre-contrast CT (HU) & 0.97 & 0.0303 & 0.343 to 0.711 & 0.0001 \\
Arterial phase (HU) & 0.53 & 0.106 & 0.651 to 0.939 & 0.7782 \\
Equilibrium phase (HU) & 0.829 & 0.0735 & 0.617 to 0.920 & 0.0001 \\
APW (\%) & 0.799 & 0.0851 & 0.617 to 0.920 & 0.0004 \\
RPW (\%) & 0.799 & 0.0851 & &
\end{tabular}

AUC, area under the curve. For APW and RPW, see Table 1. 


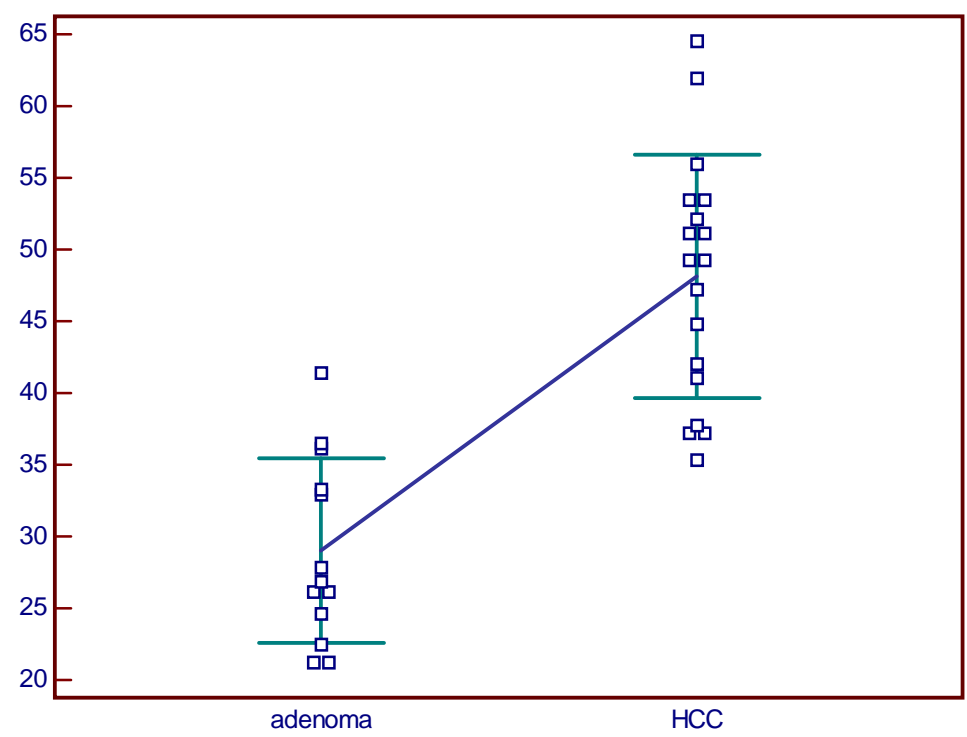

Figure 1. Scatterplot distribution of pre-contrast attenuation values of lipid-poor adenomas and metastases.

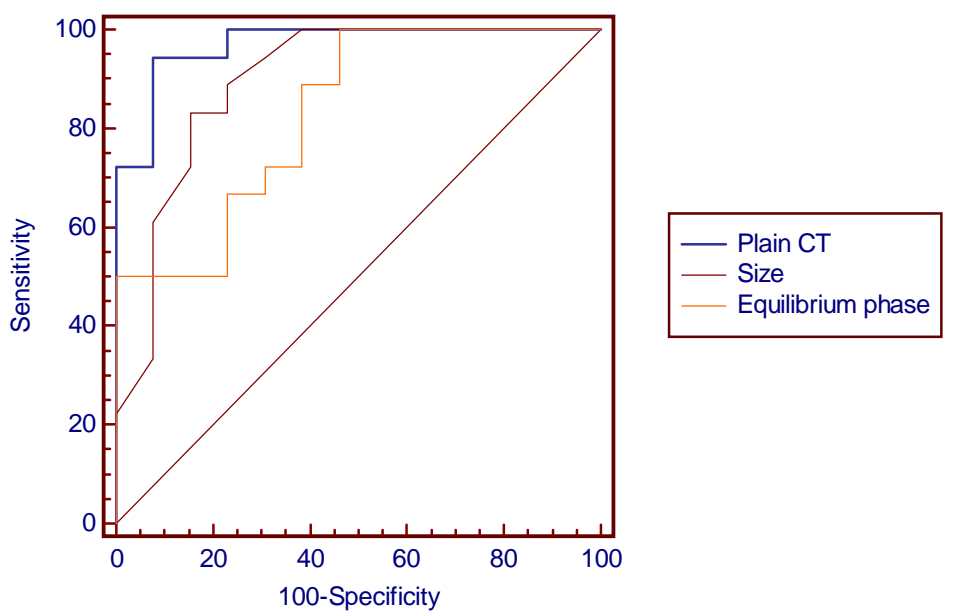

Figure 2. Comparison of receiver operating characteristic (ROC) analysis for differentiating between lipid-poor adenomas and adrenal metastases originating from HCC.

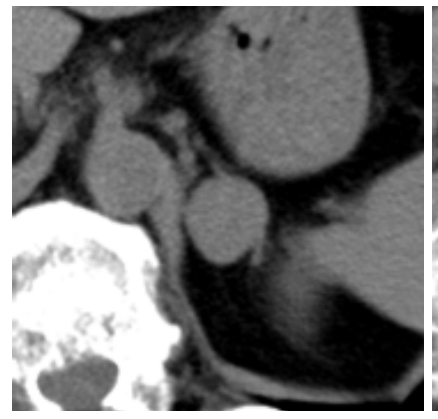

(a)

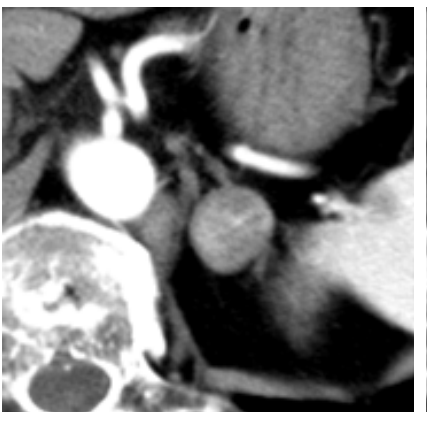

(b)

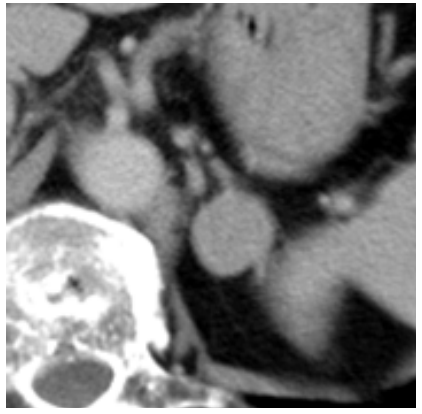

(c)

Figure 3. (a) A 69-year-old female with a typical lipid-poor adrenal adenoma. This patient underwent dynamic CT during workup for a possible hepatocellular carcinoma (HCC). There was a round, nodular lesion in the left adrenal gland with a diameter of $20 \mathrm{~mm}$. CT attenuation on the pre-contrast image (a) was $27.9 \mathrm{HU}$ (Hounsfield Units). After contrast administration, strong enhancement was observed in the nodule at the hepatic arterial phase, with $89.5 \mathrm{HU}$ (b), and some washout was observed at the late phase, with an attenuation of $71.2 \mathrm{HU}$ (c). As there was no alteration in size at a 16-month follow-up, this nodule was diagnosed as an adrenal adenoma 


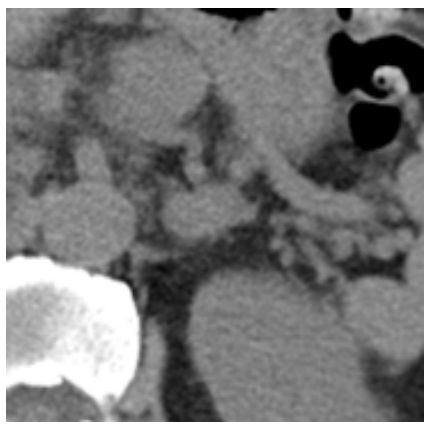

(a)

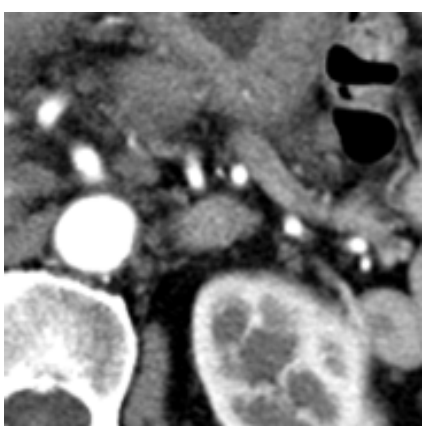

(b)

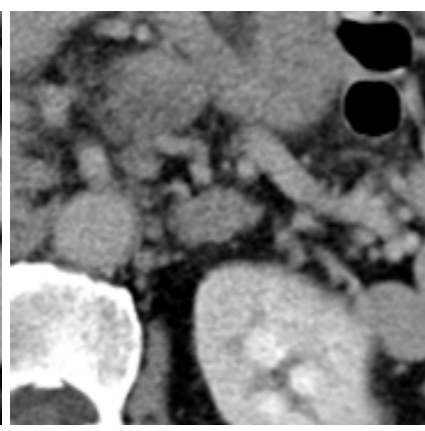

(c)

Figure 4. A 60-year-old male with an atypical lipid-poor adrenal adenoma. This patient underwent dynamic CT for follow-up imaging after resection of a hepatocellular carcinoma. There was an irregularly shaped nodular lesion (diameter: $18 \mathrm{~mm}$ ) in the left adrenal gland. CT attenuation on pre-contrast imaging was $41.4 \mathrm{HU}$ (a). After contrast administration, strong enhancement was observed in the nodule at the hepatic arterial phase, with $80.0 \mathrm{HU}$ (b), and some washout was seen at late phase, with an attenuation of $67.5 \mathrm{HU}$ (c). Although attenuation was high, even for a lipid-poor adenoma, there was no alteration in size at a follow-up of $\mathbf{1 6}$ months, and thus this nodule was diagnosed as an atypical adrenal adenoma.

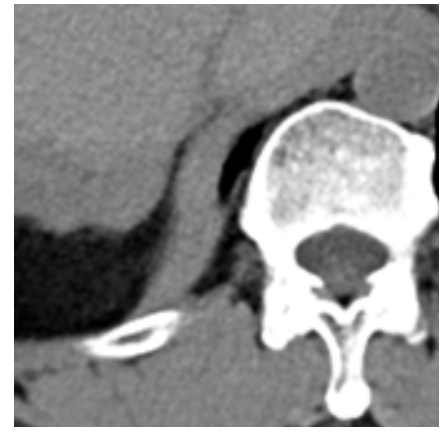

(a)

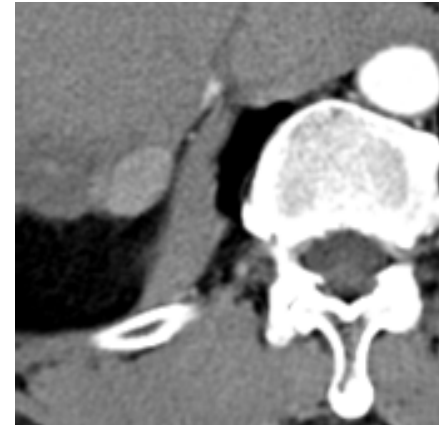

(b)

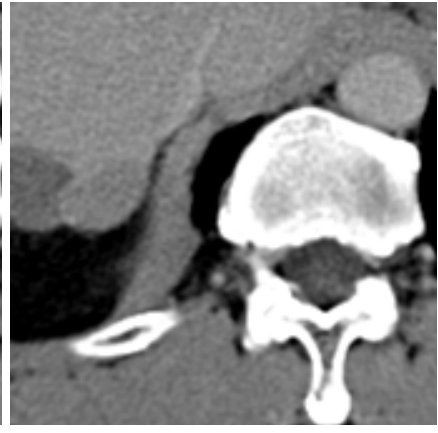

(c)

Figure 5. A 58-year-old male with a typical metastatic adrenal tumor that originated from HCC. This patient underwent dynamic CT for follow-up imaging after treatment for hepatocellular carcinoma; treatment had included resection, transarterial embolization, and percutaneous ethanol injection therapy. There was an oval, nodular lesion in the right adrenal gland with a diameter of $24 \mathrm{~mm}$. CT attenuation on the precontrast image was $53.5 \mathrm{HU}$ (a). After contrast administration, strong enhancement was observed in the nodule at the hepatic arterial phase, with $109.8 \mathrm{HU}$ (b), and some washout observed at the late phase, with an attenuation of $91.7 \mathrm{HU}$ (c). This lesion was resected and pathologically diagnosed as a metastatic adrenal tumor compatible with derivation from a moderately differentiated HCC.

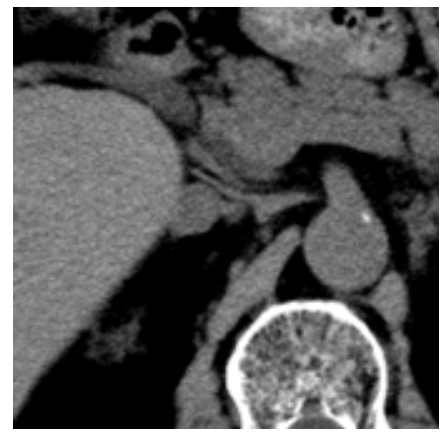

(a)

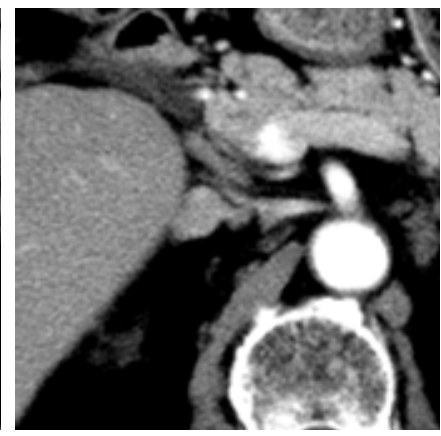

(b)

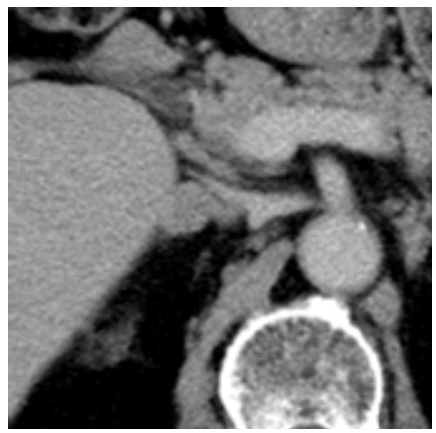

(c)

Figure 6. A 63-year-old male with an atypical metastatic adrenal tumor that had originated from HCC. This patient underwent dynamic CT for follow-up imaging after resection of a hepatocellular carcinoma. There was a nodular lesion (diameter: $22 \mathrm{~mm}$ ) in the right adrenal gland. CT attenuation on pre-contrast imaging was $35.4 \mathrm{HU}$ (a). After contrast administration, moderate enhancement was observed in the nodule at the hepatic arterial phase, with $63.7 \mathrm{HU}$ (b), and minimal washout was seen at the late phase, with an attenuation of $59.2 \mathrm{HU}$ (c). This lesion was resected and pathologically diagnosed as a metastatic adrenal tumor compatible with derivation from a moderately differentiated HCC with poorly differentiated components. 


\section{Discussion}

In this study, we attempted to characterize metastatic adrenal tumors originating from $\mathrm{HCC}$ in terms of a comparison with lipid-poor adrenal adenomas. Although we did not find any clear definition in the literature of "lipidrich" or "lipid-poor" adenomas in terms of pre-contrast CT attenuation, previous researchers have investigated attenuation threshold values of $10-25 \mathrm{HU}$ when distinguishing adenomas from other masses on pre-contrast CT; in the past decade, a sensitivity of $36 \%-89 \%$ and a specificity of $95 \%-100 \%$ was reported [15-20]. Here, we adopted a pre-contrast CT attenuation value of $20 \mathrm{HU}$ to define lipid-poor adrenal adenomas; lesions with less than $20 \mathrm{HU}$ were excluded as these were considered typical lipid-rich adenomas (or some with negative HU, myelolipomas), and lesion with $20 \mathrm{HU}$ or greater attenuation on noncontrast CT that were stable over 6 months or greater were judged to be lipid-poor adenomas. We demonstrated that lipid-poor adrenal adenomas tend to have lower attenuation characteristics than that of metastatic adrenal tumors. When the cutoff value was set at 36 $\mathrm{HU}$, sensitivity and specificity were $94.4 \%$ and $92.3 \%$, respectively. The attenuation characteristics of adrenal tumors metastasized from HCC tended to be higher than that of lipid-poor adenomas, possibly because metastatic tumors originating from $\mathrm{HCC}$ are composed primarily of moderately differentiated components that barely contain lipids.

Interestingly, we identified one case with a poorly differentiated component in a metastatic tumor under the cutoff of $36 \mathrm{HU}$ (Figure 4). As regards this particular case, differentiation from a lipid-poor adenoma was difficult due to the use of pre-contrast attenuation alone.

As expected, the RPW and APW threshold with the HCC protocol were less useful than with the 10- or 15minute protocol, due to the shorter amount of time for de-enhancement.

The majority of benign adrenal lesions are adenomas. In choosing reliable threshold values for distinguishing between benign and malignant lesions, the recognition of malignancy is paramount, even at the cost of subjecting some patients with benign lesions to biopsy. It is widely considered to be better to biopsy a few benign lesions than to miss any malignant lesion.

Several limitations to our study bear mention. This was a retrospective review of data from a relatively limited number of patients. However, it is the largest series of which we are aware, in which 18 small (less than 25 $\mathrm{mm}$ ), clinically or histopathologically diagnosed metastatic adrenal tumors originating from HCC were evaluated. In our study, histological evidence was obtained in 10 of 18 metastatic tumors, whereas the majority of the adrenal masses were not pathologically investigated and thus required imaging follow-up for characterization, according to the accepted method of classifying benign and malignant lesions in previous studies $[6,8,9,18]$. We cannot eliminate the possibility of metastases from other sites, although we found no description of the existence of any malignancies other than $\mathrm{HCC}$ in the 8 cases of malignancy examined. No pheochromocytomas or adrenal carcinomas were evaluated in our study, and therefore it remains uncertain whether or not use of this protocol will enable the differentiation of these rare tumors. When we consider that patients with chronic hepatitis and/or HCC commonly undergo regular follow-up examination using dynamic CT or MR imaging, interval increase in adrenal size may be helpful in arriving at a diagnosis of a metastatic adrenal tumor originating from HCC. It is likely that using pre-contrast CT, the diagnostic criteria presented here may increase the confidence level for a diagnosis of a metastatic tumor originating from HCC, which may be of critical importance when encountering an incidentaloma upon an initial workup for HCC, i.e., when previous images would not typically be available for comparison.

In conclusion, our results obtained with a multi-detector row $\mathrm{CT}$ protocol for HCC establish attenuation criteria for use in known HCC patients with adrenal incidentalomas; the present protocol and attenuation criteria will help optimize the differentiation between lipid-poor adenomas and metastatic adrenal masses originating from HCC in this group of patients. We therefore propose the following principles for imaging studies of HCC patients with adrenal nodules exhibiting a largest diameter of less than $30 \mathrm{~mm}$. First, all non-calcified, nonhemorrhagic adrenal lesions with a pre-contrast attenuation of greater than $35 \mathrm{HU}$ should be considered as suspicious for metastases originating from $\mathrm{HCC}$, namely, as candidates for invasive interventions such as biopsy or resection, even in the absence of a washout study with more than a 10-minute delay. Second, in adrenal lesions with a precontrast attenuation of less than $35 \mathrm{HU}$, the chance of a metastatic adrenal tumor originating from $\mathrm{HCC}$ is unlikely; however, a washout study with a delay of more than 10-minutes may be needed in such cases, because there remains the possibility of metastasis from another primary site, or that of a primary adrenal tumor such as an adrenal carcinoma, pheochromocytoma, or adrenal adenoma.

\section{REFERENCES}

[1] F. Bosch and N. Munoz, "Hepatocellular Carcinoma in the World: Epidemiologic Questions," In: E. Tabor, A. DiBisceglie and R. Purcell, Eds., Etiology, Pathology and Treatment of Hepatocellular Carcinoma in America. Advances in Applied Technology Series, Gulf, Houston, 1991, p. 35. 
[2] C. Muir, J. Waterhouse, T. Mack, J. Powell and S. Whelan, "Cancer Incidence in Five Continents," International Agency for Research on Cancer, Lyon, 1987.

[3] N. Munoz and X. Bosch, "Epidemiology of Hepatocellular Carcinoma," In: K. Okuda and K. G. Ishak, Eds., Neoplasms of the Liver, Springer, Tokyo, 1989, p. 3.

[4] K. Okuda, "Epidemiology of Primary Liver Cancer," In: T. Tobe, Ed., Primary Liver Cancer in Japan, SpringerVerlag, Tokyo, 1992, p. 3. doi:10.1007/978-4-431-68177-9 1

[5] K. Okuda, T. Ohtsuki, H. Obata, M. Tomimatsu, N. Okazaki, H. Hasegawa, Y. Nakajima and K. Ohnishi, "Natural History of Hepatocellular Carcinoma and Prognosis in Relation to Treatment. Study of 850 Patients," Cancer, Vol. 56, No. 4, 1985, pp. 918-928. doi:10.1002/1097-0142(19850815)56:4<918::AID-CNCR 2820560437>3.0.CO;2-E

[6] J. W. Marsh, I. Dvorchik, M. Subotin, V. Balan, J. Rakela, E. P. Popechitelev, V. Subbotin, A. Casavilla, B. I. Carr, J. J. Fung and S. Iwatsuki, "The Prediction of Risk of Recurrence and Time to Recurrence of Hepatocellular Carcinoma after Orthotopic Liver Transplantation: A Pilot Study," Hepatology, Vol. 26, No. 2, 1997, pp. 444-450. doi:10.1002/hep.510260227

[7] T. Nakashima, K. Okuda, M. Kojiro, A. Jimi, R. Yamaguchi, K. Sakamoto and T. Ikari, "Pathology of Hepatocellular Carcinoma in Japan. 232 Consecutive Cases Autopsied in Ten Years," Cancer, Vol. 51, No. 5, 1983, pp. 863-877.

doi:10.1002/1097-0142(19830301)51:5<863::AID-CNCR 2820510520>3.0.CO;2-D

[8] L. Barzon, N. Sonino, F. Fallo, G. Palu and M. Boscaro, "Prevalence and Natural History of Adrenal Incidentalomas," European Journal of Endocrinology, Vol. 149, No. 4, 2003, pp. 273-285. doi:10.1530/eje.0.1490273

[9] Mayo-Smith WW, Boland GW, Noto RB and Lee MJ, "State-of-the-Art Adrenal Imaging." Radiographics, Vol. 21, 2001, pp. 995-1012.

[10] M. Korobkin, F. J. Brodeur, G. G. Yutzy, I. R. Francis, L. E. Quint, N. R. Dunnick and E. A. Kazerooni, "Differentiation of Adrenal Adenomas from Nonadenomas Using CT Attenuation Values," AJR American Journal of Roentgenology, Vol. 166, No. 3, 1996, pp. 531-536.

[11] E. M. Caoili, M. Korobkin, I. R. Francis, R. H. Cohan, J. F. Platt, N. R. Dunnick and K. I. Raghupathi, "Adrenal Masses: Characterization with Combined Unenhanced and Delayed Enhanced CT," Radiology, Vol. 222, No. 3, 2002, pp. 629-633. doi:10.1148/radiol.2223010766
[12] W. W. Mayo-Smith, "CT Characterization of Adrenal Masses,” Radiology, Vol. 226, No. 1, 2003, pp. 289-290. doi:10.1148/radiol.2261020528

[13] S. Katyal, J. H. Oliver, M. S. Peterson, J. V. Ferris, B. S. Carr and R. L. Baron, "Extrahepatic Metastases of Hepatocellular Carcinoma," Radiology, Vol. 216, No. 3, 2000 , pp. 698-703.

[14] T. Ichikawa, S. M. Erturk and T. Araki, "Multiphasic Contrast-Enhanced Multidetector-Row CT of Liver: Contrast-Enhancement Theory and Practical Scan Protocol with a Combination of Fixed Injection Duration and Patients' Body-Weight-Tailored Dose of Contrast Material,' European Journal of Radiology, Vol. 58, No. 2, 2006, pp. 165-176. doi:10.1016/j.ejrad.2005.11.037

[15] D. H. Szolar, M. Korobkin, P. Reittner, A. Berghold, T. Bauernhofer, H. Trummer, H. Schoellnast, K. W. Preidler and H. Samonigg, "Adrenocortical Carcinomas and Adrenal Pheochromocytomas: Mass and Enhancement Loss Evaluation at Delayed Contrast-Enhanced CT," Radiology, Vol. 234, No. 2, 2005, pp. 479-485. doi:10.1148/radiol.2342031876

[16] S. H. Park, M. J. Kim, J. H. Kim, J. S. Lim and K. W. Kim, "Differentiation of Adrenal Adenoma and Nonadenoma in Unenhanced CT: New Optimal Threshold Value and the Usefulness of Size Criteria for Differentiation," Korean Journal of Radiology, Vol. 8, No. 4, 2007, pp. 328- 335. doi:10.3348/kjr.2007.8.4.328

[17] F. Ctvrtlik, M. Herman, V. Student, V. Ticha and J. Minarik, "Differential Diagnosis of Incidentally Detected Adrenal Masses Revealed on Routine Abdominal CT," European Journal of Radiology, Vol. 69, No. 2, 2009, pp. 243-252. doi:10.1016/j.ejrad.2007.11.041

[18] C. S. Pena, G. W. Boland, P. F. Hahn, M. J. Lee and P. R. Mueller, "Characterization of Indeterminate (Lipid-Poor) Adrenal Masses: Use of Washout Characteristics at Contrast-Enhanced CT," Radiology, Vol. 217, No. 3, 2000, pp. 798-802.

[19] G. M. Israel, M. Korobkin, C. Wang, E. N. Hecht and G. A. Krinsky, "Comparison of Unenhanced CT and Chemical Shift MRI in Evaluating Lipid-Rich Adrenal Adenomas," AJR American Journal of Roentgenology, Vol. 183, No. 1, 2004, pp. 215-219.

[20] B. K. Park, B. Kim, K. Ko, S. Y. Jeong and G. Y. Kwon, "Adrenal Masses Falsely Diagnosed as Adenomas on Un-Enhanced and Delayed Contrast-Enhanced Computed Tomography: Pathological Correlation," European Radiology, Vol. 16, No. 3, 2006, pp. 642-647. doi:10.1007/s00330-005-0017-0 